\title{
Photoproduction of relativistic positronium atoms on extended targets
}

\author{
S.R.Gevorkyan! \\ Laboratory of Nuclear Problems, \\ Joint Institute for Nuclear Research, \\ Dubna, Moscow Region, 141980, Russia \\ S.S.Grigoryan \\ Yerevan Physics Institute, \\ Yerevan,375036, Armenia
}

\begin{abstract}
It is shown that the yield of relativistic positronium atoms in photoproduction on extended targets is much larger than usually accepted.The cause of such difference is transparent. At high energies, when the positronium atom formation time $\tau_{f}$ becomes greater than the target length $\mathrm{L}$, it is the free $e^{+} e^{-}$pair that propagates through the target and the formation of positronium atoms take place out of the target.In the framework of the light-cone technique we consider the interaction of $e^{+} e^{-}$pair with the screened Coulomb field of atoms in matter and obtain the analytical expressions for relativistic positronium atoms photoproduction cross sections on extended targets. The relations to free pair photoproduction on extended targets are discussed.
\end{abstract}

\section{Introduction}

Long ago it was predicted [1, 2] that the probability for observing the relativistic positronium atom after its passage through a thin layer of matter is inversely proportional to the layer thickness L. This effect takes place when $\mathrm{L}$ is much smaller than the characteristic positronium internal time $\tau_{n}=\frac{1}{\varepsilon_{n}}$ Lorentz dilated in laboratory system

$$
\tau_{f}=\gamma \tau_{n} \gg L
$$

Here $\varepsilon_{n}$ is the positronium binding energy for the state with the principal quantum number $\mathrm{n} ; \gamma=E / 2 m$ is the positronium Lorentz factor;E,m are the positronium energy and the electron mass. (Throughout we use units for which $c=\hbar=1$ ) This time can be interpreted as the positronium atom formation time [3]. Such behavior considerably deviates from the canonical (exponential) law, which is usual for the survival probabilities in matter. The physical reason for this difference is the possibility of a positronium atom fluctuating to different excited states during its passage through the slab of matter [4, 5].From the point of view of positronium internal structure the deviation from the exponential low is a direct consequence of positronium neutrality. The neutral system interacts with matter as a dipole,so its total cross section with the target atoms at small transverse separation $\mathrm{r}$ between constituents of the pair behaves like $\sigma \sim r^{2}$. To obtain the survival probability

\footnotetext{
${ }^{1}$ On leave of absence from Yerevan Physics Institute
} 
for the $e^{+} e^{-}$pair one has to average the survival probability with definite transverse separation $\mathrm{r}$ (the survival probability for such a state obeys the usual exponential law) using the positronium wave function, which leads to behavior different from the canonical one [2].

A similar effect was predicted in Quantum Chromodynamics(QCD), where it is known as color transparency.In QCD it is a case of color neutrality of hadrons.At certain conditions (deep inelastic scattering, hard processes)the created complex system traversing a nucleus undergoes a substantially smaller absorption than a usual hadron. This is one of the most salient QCD predictions, which has been widely investigated during the last years [6]. Unfortunately, up to now there has been no unique experimental check on the existence of this effect and only future efforts can shed light on this fundamental property of QCD.Thus the investigation of the passage of relativistic positronium atoms through matter is an important and fundamental task.

Relativistic positronium atoms were observed at Protvino [7] by using the neutral pion decay with subsequent transition of one of the photons into $e^{+} e^{-}$pair, among which positronium atoms were detected.Unfortunately, the low intensity of such beams and some experimental problems did not allow to investigation of the predicted transparency for passage of relativistic positronium atoms through matter. An exciting possibility of creating sufficiently intense beams of relativistic positronium atoms is offered by the photoproduction of $e^{+} e^{-}$pairs in the Coulomb field of atoms(Bethe-Heitler process).According to the estimates [3, \&] this process can be a real source of relativistic positronium atoms. The cross sections for photo- and electroproduction of positronium atoms in the Coulomb field are known in all orders in fine structure constant and for any momentum transfer [9]. The goal of the present paper is to investigate the photoproduction of positronium atoms on extended targets and effects appearing during their passage through medium.This is not only interesting from the theoretical point of view, but also can be useful for future experiments.

\section{The positronium photoproduction amplitude}

The amplitude of the process $\gamma+T \rightarrow P s+T$, where $\mathrm{T}$ is an extended target and Ps the positronium atom, can be obtained by the powerful light-cone technique developed for quantum electrodynamic processes at high energies [10]. In the light-cone formalism the amplitude for $e^{+} e^{-}$pair production is calculated and then projected onto a certain state(Ps in our case).In the framework of this approach the amplitude for Ps photoproduction is

$$
F=\frac{i}{2 \pi} \int e^{i \vec{Q} \vec{R}} \psi_{p}(\vec{r}, \alpha) \Phi_{f} \Gamma\left(\vec{r}, \vec{R}, \vec{s}_{1} \ldots \vec{s}_{N}\right) \Phi_{i} \psi_{\gamma}(\vec{r}, \alpha) d^{2} r d^{2} R d \alpha d^{2} s_{1} \ldots d^{2} s_{N}
$$

where $\vec{Q}$ is the momentum transferred to the target; $\vec{R}, \vec{s}_{i}$ are the photon impact factor and the transverse coordinates of atoms in the target; $\psi_{p}(\vec{r}, \alpha)$ is the light-cone positronium wave function in the mixed $(\vec{r}, \alpha)$ representation, where $\alpha$ is the fraction of the photon light-cone momentum carried by the electron and $\vec{r}$ is the transverse separation in the $e^{+} e^{-}$pair; $\psi_{\gamma}(\vec{r}, \alpha)$ is the wave function (non-normalized) of the $e^{+} e^{-}$fluctuation of the photon. $\Phi_{i(f)} \equiv \Phi_{i(f)}\left(\vec{s}_{1}, \vec{s}_{2} \ldots \vec{s}_{N}\right)$ are the distributions of atoms in the target before and 
after photoproduction.At last,

$$
\Gamma=1-\exp \left(i \sum\left[\chi\left(\vec{s}_{k}-\vec{R}-\frac{\vec{r}}{2}\right)-\chi\left(\vec{s}_{k}-\vec{R}+\frac{\vec{r}}{2}\right)\right]\right)
$$

where the eikonal phase $\chi(\vec{\rho})=-\int V(\vec{\rho}, z) d z$ and $V(\vec{\rho}, z)$ is the Coulomb potential of the target atoms.Amplitude (2) is normalized according to the condition:

$$
\frac{d \sigma}{d^{2} Q}=|F|^{2}
$$

This structure of the amplitude is the direct result of the space-time picture of the photoproduction processes at high energies, which can be considered as proceeding in three steps.At the first step the high energy photon splits into an $e^{+} e^{-}$pair. The lifetime of such fluctuation is determined by the so-called coherence length $l_{c}$ (an excellent review on this subject and related topics can be found in [11]). When the coherence length becomes larger than the target length $\mathrm{L}$

$$
l_{c}=\frac{E}{2 m^{2}} \geq L
$$

this transition takes place before the target. The next step is the Coulomb interaction of the $e^{+} e^{-}$pair with atoms of the target, which is determined by (3).As a result of Lorentz dilation, the pairs at high energies pass through the slab of matter with unchanged transverse separation $r$. The last step is the process of positronium atom formation with the lifetime $\tau_{f}$ determined by (1). Since the binding energies in positronium atoms are small(for example the energy of the Ps ground state is $\varepsilon_{1}=6.8 \mathrm{ev}$ ), the positronium formation length $l_{f}=c \tau_{f}$ is always larger than the coherence length. So if condition (5) is fulfilled,the formation of a positronium atom always takes place far beyond the target.

\section{The light-cone wave functions}

The probability amplitude for a photon to interact with the target as an $e^{+} e^{-}$pair $\psi_{\gamma}(\vec{r}, \alpha)$ is well known [10, 12]. We will use this amplitude for the case when the electron and the positron possess the same fraction of the light-cone momentum $\alpha=1-\alpha=1 / 2$. The reason is that this amplitude is a slowly varying function of $\alpha$ in comparison with the positronium wave function, which is a sharp function of $\alpha$ and peaks at $\alpha=1 / 2$. To show this, let us derive the positronium wave function in the mixed $\vec{r}, \alpha$ representation. The positronium wave function in positronium rest frame is well known [13]

$$
\psi_{p}(\vec{q})=\frac{8 \sqrt{\pi a_{B}{ }^{3}}}{\left(1+a_{B}^{2} q^{2}\right)^{2}}
$$

Here $a_{B}=\frac{2}{m \alpha_{e m}}$ is the Ps radius, $\alpha_{e m}=1 / 137$ is the fine structure constant and $\vec{q}$ is the relative momentum in Ps. To get the Ps wave function in the mixed $(\vec{r}, \alpha)$ representation, we carry on the following standard procedure [14. In the Ps rest frame (the $e^{+} e^{-}$center of mass system) the longitudinal component of the relative momentum $q_{z}$ is connected with the invariant mass of the $e^{+} e^{-}$pair $\mathrm{M}$ by a simple relation $q_{z}=(\alpha-1 / 2) M$.Using the relation between the invariant mass and the transverse component of the relative 
momentum $\overrightarrow{q_{t}}: M^{2}=\frac{q_{t}^{2}+m^{2}}{\alpha(1-\alpha)}$ and taking the two-dimensional Fourier transform of (6), we obtain:

$$
\psi_{p}(r, \alpha)=\frac{2 r}{\pi a_{B}} \sqrt{\frac{m}{a_{B}}} \frac{\eta^{5 / 4}}{\sqrt{m^{2} a_{B}^{2}-\eta\left(m^{2} a_{B}^{2}-1\right)}} K_{1}\left(\frac{r \sqrt{m^{2} a_{B}^{2}-\xi\left(m^{2} a_{B}^{2}-1\right)}}{a_{B}}\right)
$$

where $\eta=4 \alpha(1-\alpha) ; K_{1}(x)$ is the modified Bessel function (MacDonald function) of first order. The peak of this expression is for symmetric pairs $(\eta=1)$ and quickly decreases for asymmetric ones. On the other hand, the probabilistic amplitude for a photon to be in the $e^{+} e^{-}$state $\psi_{\gamma}(\vec{r}, \alpha)$ is determined by the modified Bessel functions [12] $K_{0}(m r)$ and $K_{1}(m r)$, whose asymptotic behavior is $K_{0}(x), K_{1}(x) \sim \sqrt{\frac{\pi}{2 x}} \exp (-x)$. Thus the essential value of $r$ in (2) is $r \leq \frac{1}{m}$. As to positronium wave function (7), its behavior is determined by atomic dimensions so that $r \leq \frac{1}{m \alpha_{e m}}$. This allows us to factor the positronium wave function outside the integration in (2) at $\mathrm{r}=0$ and integrate it over $\alpha$.As a result, we replace the Ps wave function in (2) by the value $m\left(\frac{\alpha_{e m}}{2}\right)^{3 / 2}$

In what follows we need to know the product of photon wave functions $\psi_{\gamma}(\vec{r}, \alpha)$ at equal fraction of the light-cone momentum of the photon carried by the constituents of the pair and at a different separation in the transverse plane.Considering the results of [12], it is

$$
\psi_{\gamma}(\vec{r}) \psi_{\gamma}(\vec{r} \prime)=\frac{2 \alpha_{e m} m^{2}}{(2 \pi)^{2}}\left(K_{0}(m r) K_{0}(m r \prime)+\frac{\vec{r} \vec{r} \prime}{2 r r \prime} K_{1}(m r) K_{1}(m r \prime)\right)
$$

\section{The probability of positronium atom production from an extended target}

In order to obtain the differential cross section for the process $\gamma+T \rightarrow P s+T$ we use the completeness condition for the final distribution of atoms in the target:

$$
\sum_{f} \Phi_{f}\left(\vec{s}_{1} \ldots \vec{s}_{N}\right) \Phi_{f}\left(\vec{s}_{1} \ldots \vec{s}_{N}\right)=\delta\left(\vec{s}_{1}-\vec{s}_{1}\right) \ldots \delta\left(\vec{s}_{N}-\vec{s}_{N}\right)
$$

Furthermore, in an amorphous target the atoms are arranged chaotically and hence their position is equiprobable, so that for the initial distribution

$$
\left|\Phi_{i}\left(\vec{s}_{1} \ldots \vec{s}_{N}\right)\right|^{2}=\left(\frac{1}{S}\right)^{N}
$$

Here $\mathrm{S}$ is the target surface; $\mathrm{N}=\mathrm{nSL}$ is the number of atoms in the target, and $\mathrm{n}$ is the number of atoms in the unit volume. Using this relations for the distributions of atoms in the target and expressions (2)-(4), we obtain for the differential cross section

$$
\begin{gathered}
\frac{d \sigma}{d^{2} Q}=\frac{m^{2} \alpha_{e m}^{3} S}{8(2 \pi)^{2}} \int d^{2} b d^{2} r d^{2} r e^{i \vec{Q} \vec{b}} \psi_{\gamma}(\vec{r}) \psi_{\gamma}(\vec{r} \prime) E(\vec{r}, \vec{r} \prime, \vec{b}) \\
E(\vec{r}, \vec{r} \prime, \vec{b})=1+e^{-n L \int d^{2} \rho\left(1-\cos \left(\chi-\chi^{\prime}\right)\right)}-e^{-n L \int d^{2} \rho(1-\cos \chi)}-e^{-n L \int d^{2} \rho\left(1-\cos \chi^{\prime}\right)} \\
\chi=\chi\left(\vec{\rho}-\frac{\vec{r}}{2}\right)-\chi\left(\vec{\rho}+\frac{\vec{r}}{2}\right) ; \chi^{\prime}=\chi\left(\vec{b}+\vec{\rho}-\frac{\vec{r} \prime}{2}\right)-\chi\left(\vec{b}+\vec{\rho}+\frac{\vec{r} \prime}{2}\right)
\end{gathered}
$$


As was mentioned above, the effective size of the pair is determined by the asymptotic behavior of the modified Bessel function(see (8)) so that $r, r^{\prime} \leq \frac{1}{m}$. On the other hand, in the interaction of the pair with atoms the atomic dimensions are essential. In the case of screened Coulomb potential $R_{a} \sim \frac{1}{m \alpha_{e m} Z^{1 / 3}} \sim 5 * 10^{-9} Z^{-1 / 3} \mathrm{~cm}$ they are much larger than $\frac{1}{m}$. This allows one to expand the cosine in (12) and to restrict oneself to the lowest order of this expansion.In this approximation the integrals in (12) are

$$
f(r)=\frac{1}{2} \int \chi^{2} d^{2} \rho=\int d^{2} q \frac{d \sigma}{d^{2} q}\left(1-J_{0}(q r)\right) ; \int \chi^{*} \chi^{\prime} d^{2} \rho=f\left(\frac{|\vec{r}+\vec{r} \prime|}{2}\right)-f\left(\frac{|\vec{r}-\vec{r} \prime|}{2}\right)
$$

where $\frac{d \sigma}{d^{2} q}=\frac{4 \alpha_{e m}^{2} Z^{2}}{q^{4}}\left(1-F_{a}(q)\right)^{2}$ is the differential cross section for interaction of an electron with a single atom and $F_{a}(q)$ is the atomic formfactor.In what follows we will use a simplified parameterization for the Thomas-Fermi-Moliere formfactor

$$
F_{a}(q)=\frac{1}{1-q^{2} / \Lambda^{2}} ; \Lambda=\frac{Z^{1 / 3} m}{111}
$$

which allows the analytical expressions for $\mathrm{f}(\mathrm{r})$ to be obtained.Substituting this parameterization in (13) and keeping in mind that $\mathrm{r}$ and $r /$ are not too large,we obtain by straightforward calculations that

$$
f(r)=\xi\left(\frac{r}{2}\right)^{2} ; \xi=8 \pi\left(\alpha_{e m} Z\right)^{2}\left[\ln \left(222 Z^{-1 / 3}\right)+\frac{1-2 \gamma}{2}\right]
$$

where $\gamma=0.577$ the Euler constant. With this relation the expression (12) can be written as

$$
E(\vec{r}, \vec{r} \prime, \vec{b})=1-e^{\frac{-n \xi L r^{2}}{4}}-e^{-n \xi L\left(\vec{b}-\frac{\vec{r} \prime}{2}\right)^{2}}+e^{-n \xi L\left(\vec{b}+\frac{\vec{r}-\vec{r} \prime}{2}\right)^{2}}
$$

Thus, expressions (8),(11) and (15) allow one to calculate the differential cross section for the process of relativistic positronium photoproduction on extended targets for any momentum transfer.

For numerical calculation it is more suitable to deal with the total cross section of Ps photoproduction.Integrating (11) over the momentum transfer $\vec{Q}$ we get

$$
\begin{gathered}
\sigma=\int \frac{d \sigma}{d^{2} Q} d^{2} Q=\frac{\alpha_{e m}^{4} m^{4} S}{8 \pi} \int\left[K_{0}(m r) K_{0}(m r \prime)+\frac{\cos \theta}{2} K_{1}(m r) K_{1}(m r \prime)\right] E(r, r \prime, d \theta) r d r r / d r \prime d \theta \\
E(r, r \prime, \theta)=1-e^{\frac{-n \xi L r^{2}}{4}}-e^{\frac{-n \xi L r^{2}}{4}}+e^{\frac{-n \xi L\left(r^{2}+r \prime^{2}\right)}{4}} e^{\frac{n \xi L r r \cos \theta}{2}}
\end{gathered}
$$

Taking advantage of the integral representation for modified Bessel functions [15]

$$
K_{\nu}(z)=\frac{1}{2}\left(\frac{z}{2}\right)^{\nu} \int_{0}^{\infty} \frac{e^{-t-\frac{z^{2}}{4 t}} d t}{t^{\nu+1}}
$$

one can calculate the integrals entering into(16) with the following results

$$
\begin{gathered}
\int K_{0}(r) e^{\frac{-a r^{4}}{2}} r d r=-\frac{1}{a} e^{\frac{1}{a}} \operatorname{Ei}\left(-\frac{1}{a}\right) \\
\int K_{0}(r) K_{0}(r \prime) I_{0}\left(\frac{a r r \prime}{2}\right) e^{-\frac{a\left(r^{2}+r^{2}\right)}{4}} r d r r \prime d r \prime=\frac{1}{a}+\frac{e^{\frac{1}{a}}}{a^{2}} \operatorname{Ei}\left(-\frac{1}{a}\right)
\end{gathered}
$$




$$
\int K_{1}(r) K_{1}(r \prime) I_{1}\left(\frac{a r r \prime}{2}\right) e^{-\frac{a\left(r^{2}+r^{2}\right)}{4}} r d r r \prime d r \prime=-\frac{1}{a}-e^{\frac{1}{a}} \frac{(1+a)}{a^{2}} \operatorname{Ei}\left(-\frac{1}{a}\right)
$$

Here $I_{0}, I_{1}$ are the Bessel functions of the imaginary argument and $\operatorname{Ei}(-x)=-\int_{x}^{\infty} \frac{e^{-t}}{t} d t$ is the exponential integral [15. Finally, the total cross section of positronium atom photoproduction from an extended target takes the form

$$
\sigma=\frac{\alpha_{e m}{ }^{4} S}{4}\left[1+\frac{x}{2}+\frac{x(3+x)}{2} e^{x} E i(-x)\right]
$$

with $x=\frac{m^{2}}{n \xi L}$. For a thin $\operatorname{target}(x \gg 1)$ using the asymptotic behavior of $\operatorname{Ei}(-x)$ [15 one can write at once:

$$
\begin{gathered}
\sigma=n L S \frac{\alpha_{e m}^{4} \xi}{8 m^{2}}=N \sigma_{0} \\
\sigma_{0}=\frac{\pi Z^{2} \alpha_{e m}^{6}}{m^{2}}\left[\ln \left(222 Z^{-1 / 3}\right)+\frac{1-2 \gamma}{2}\right]
\end{gathered}
$$

so that $\sigma_{0}$ is the total cross section of positronium photoproduction on a single atom [9]. Thus,in the limiting case of thin targets the yield of positronium atoms is proportional to the target length.

For a thick target $(x \ll 1)$,expanding the exponential integral in $(22)$ we get

$$
\sigma=\frac{\alpha_{e m}^{4} S}{4}\left(1-\frac{3 m^{2} \ln \frac{n \xi L}{m^{2}}}{2 n \xi L}\right)
$$

As can be seen from this expression the absorption of pairs in the target take place in accordance with the power low.

The yield of Ps in photo- and electroproduction on extended targets is usually estimated by a standard expression [16, 17] obtained in the framework of the optical model

$$
\sigma_{\text {opt }}=\sigma_{0} S \frac{1-e^{-n \sigma_{d} L}}{\sigma_{d}}
$$

where $\sigma_{0}$ is the total cross section for positronium photoproduction on atom(expression (24)) and $\sigma_{d}$ is the cross section for positronium dissociation on a single atom.This expression coincides with our result (22) only in the limit of extremely thin targets $(L \rightarrow 0)$ and sharply disagrees with it as $\mathrm{L}$ increases. To illustrate this difference we calculated the ratio of the total cross sections given by expressions (22) and (26) $R=\frac{\sigma}{\sigma_{\text {opt }}}$ for the copper as a target.For the positronium dissociation cross section in the Coulomb field of an atom we use the expressions from [3] $\sigma_{d}=0.94 Z^{1.24} 10^{-19} \mathrm{~cm}^{2}$. The number of atoms in the unit volume is $n=\frac{\rho N_{A}}{A}$ where $\rho$ is the target density and $N_{A}$ is Avogadro constant. The quantity $\xi$ was calculated by expression (14). As was mentioned above $\mathrm{R}$ is equal to one only for extremely small L.For $L=1 \mu m R \approx 25$ and for $L=10 \mu m$ this ratio increase to $R \approx 375$. Thus, the difference between the predictions of the present work and the expression explored in the literature is great and considerably increases with increasing $\mathrm{L}$. The cause of such difference is transparent.Expression (26) corresponds to the physical picture when the positronium atom produced in the target and its formation takes place instantly.As can be seen from inequality (1) for positronium formation time this picture can be correct only for extremely thin targets or rather low energies.In our approach the relativistic positronium atoms are created far behind the target and it is a free $e^{+} e^{-}$pair that goes through the target and interacts with matter. 


\section{The relation between the photoproduction of positro- nium and free pairs}

It is well known that the cross section for positronium photoproduction on atom is uniquely related to that for free $e^{+} e^{-}$pairs [8]. To find a similar correspondence in the case of extended targets we have to know the cross section for the process $\gamma+T \rightarrow e^{+} e^{-}+T$. The amplitude for this process in the mixed $(r, \alpha)$ representation can be obtained by the same technique from [10]

$$
F=\frac{i}{(2 \pi)^{2}} \int e^{i \vec{q} \vec{r}+i \vec{Q} \vec{R}} \psi_{\gamma}(\vec{r}, \alpha) \Phi_{f} \Gamma\left(\vec{r}, \vec{R}, \vec{s}_{1} \ldots \vec{s}_{n}\right) \Phi_{i} d^{2} r d^{2} R d^{2} s_{1} \ldots d^{2} s_{n}
$$

The notation is the same as in expression (2). The new is the relative momentum of the pair $\vec{q}=\alpha \vec{p}_{1}-(1-\alpha) \vec{p}_{2}$, where $\vec{p}_{1}, \vec{p}_{2}$ are the electron and positron momenta. The differential cross section for free pair photoproduction is given by relation

$$
\frac{d \sigma}{d \alpha d^{2} Q d^{2} q}=|F|^{2}
$$

Using the same technique as in the positronium case we get the following expression for the unbound pair photoproduction differential cross section:

$$
\frac{d \sigma}{d \alpha d^{2} q d^{2} Q}=\frac{S}{2 \pi^{2}} \int e^{i \vec{q}(\vec{r}-\vec{r} \prime)+i \vec{Q} \vec{b}} \psi_{\gamma}(\vec{r}, \alpha) \psi_{\gamma}(\vec{r} \prime, \alpha) E(\vec{r}, \vec{r} \prime, \vec{b}) d^{2} r d^{2} r \prime d^{2} b
$$

Comparing this expression with (11) we find the relation between the differential cross sections for photoproduction of positronium atoms on an extended targets and the same quantity for free pairs

$$
\frac{d \sigma}{d^{2} Q}=(2 \pi)^{2}\left|\int \psi_{p}(0, \alpha) d \alpha\right|^{2} \frac{d \sigma}{d \alpha d^{2} Q d^{2} q}(\alpha=1 / 2, \vec{q}=0)
$$

Thus, the cross section for Ps photoproduction on an extended target can be expressed in terms of the differential cross section for free pair production at the equal longitudinal momenta of the electron and the $\operatorname{positron}(\alpha=1-\alpha=0.5)$ and the zero relative momentum and positronium atom light-cone wave function at zero transverse separation.

Integrating the differential cross section for unbound pair photoproduction (29) over the transverse momenta $\vec{Q}$, we get

$$
\frac{d \sigma}{d \alpha}=2 S \int\left(1-e^{-\frac{n a L r^{2}}{4}}\right)\left|\psi_{\gamma}(\vec{r}, \alpha)\right|^{2} d^{2} r
$$

The physical meaning of this expression is transparent.For a thin target $(L \rightarrow 0)$ the cross section is determined by the total number of atoms in the target and the Bethe-Heitler cross section on atom. On thick target the production of unbound pairs is suppressed being the result of destructive interference of photoproduction amplitudes from different atoms known as the Landau-Pomeranchuk effect [11. 


\section{Conclusions}

In summary,we have studied the photoproduction of bound and free $e^{+} e^{-}$pairs on an extended target at high energies. We obtained analytical expressions for the differential and total cross sections of these processes on extended targets and relation between the bound and free pair yields.Comparing our result for positronium photoproduction on extended targets with the predictions obtained in the naive optical model we show a large difference between them.Even for the targets with several micrometers length our predictions are two orders higher then generally accepted and rapidly grows with increasing the target length. This open a nice opportunity to create relativistic positronium atom beams at electron accelerators.

The extension of the above results for the case of virtual photons is a simple task.For this one has to replace the mass $m$ in the arguments of the Bessel functions in (8) according to prescription [10 by $m^{2} \rightarrow m^{2}+Q^{2}$ where $\mathrm{Q}$ is the photon virtuality.

\section{Acknowledgements}

S.R.G.would like to thank A.V.Tarasov for useful discussions.This work was supported by INTAS within Project No.INTAS-97-3455.

\section{References}

[1] L.L.Nemenov, Yad.Fiz.34,1308 (1981)

[2] V.L.Lyuboshits and M.I.Podgoretsky, JETP 81,1556 (1981)

[3] L.L.Nemenov Yad.Fiz. 51,444 (1990)

[4] B.G.Zakharov, Yad.Fiz.46,148 (1987)

[5] A.S.Pak and A.V.Tarasov, Yad.Fiz. 45,145 (1987)

[6] P.Jain,B.Pire and J.Ralston Phys.Rep. 273(1996)67

[7] L.G.Afanasyev et al. Yad.Fiz.50,7(1989)

[8] V.L.Lyuboshits Yad.Fiz. 45,1099(1987)

[9] S.R.Gevorkyan,E.A.Kuraev,A.V.Tarasov,A.Schiller and V.G.Serbo Phys.Rev A58,4856 (1998)

[10] J.M.Bjorken,J.B.Kogut and D.E.Soper, Phys.Rev.D3,1382(1971)

[11] S.Klein Rev.Mod.Phys.71,1501 (1999)

[12] B.Z.Kopeliovich,A.Schafer and A.V.Tarasov,Phys.Rev.D62,054022(2000)

[13] A.I.Akhieser and V.B.Berestetski,Quantum Electrodynamics (Interscience,New York,1965) 
[14] W.Jaus,Phys.Rev.D44,2851(1991)

[15] I.S.Gradshtein and I.M.Ryzhik, Tables of Integrals,Series and Products (Moscow,Nauka,1971)

[16] A.A.Akhundov,D.Yu.Bardin and L.L.Nemenov, Yad.Phys.27,1542(1978)

[17] L.S.Dulyan and Ar.M.Kotzinian,Yad.Phys.37,137(1983) 